\title{
Investigación como estrategia pedagógica apoyada en tic en la Institución Educativa Departamental Macondo
}

\section{Research as a pedagogical strategy supported by tic in the Macondo Departmental Educational Institution}

DOI: http://dx.doi.org/10.17981/cultedusoc.9.3.2018.92

Artículo de investigación. Fecha de recepción: 15/06/2018. Fecha de aceptación: 27/11/2018

Luz Padilla Romero';

Alexander Gutiérrez-Vergel; América Ortega-Cantillo; Ana Barrios-Cervantes; Arnaldo Tilano-Marriaga; Augusto Gutiérrez; Edwin Zúñiga-Vega;

Elizabeth Monsalvo-Martínez; Ernesto Urrea-Garrido; Inés Mercado-Meyer; Ingri Escobar-Álvarez; Jairo Morales-Jiménez; Joaquín Coronel;

José Espinoza-Hernández; Karina Diazgranados-Sánchez; Katiana Pineda-Castillo; Liseth Márquez Benítez; Liliana Galue-Bermúdez; Lucas Romero-Matos;

Ludys Barrios-Cervantes; Luz Padilla-Romero;

Ninfa Salcedo-Algarin y Priscila Pacheco-Martínez ${ }^{2}$

Institución educativa Macondo del municipio de la zona Bananera (Colombia)

lucipa68@hotmail.com

Para citar este artículo:

Padilla, L., Gutiérrez-Vergel, A., Ortega-Cantillo, A., Barrios-Cervantes, A., Tilano-Marriaga, A., Gutiérrez, A., Zúñiga-Vega, E., Monsalvo-Martínez, E., Urrea-Garrido, E., Mercado-Meyer, I., Escobar-Álvarez, I., Morales-Jiménez, J.,; Coronel, J., EspinozaHernández, J., Diazgranados-Sánchez, K., Pineda-Castillo, K., Márquez Benítez, L., Galue-Bermúdez, L., Romero-Matos, L., BarriosCervantes, L., Padilla-Romero, L., Salcedo-Algarin. N. y, Pacheco-Martínez, P. (2018). Investigación como estrategia pedagógica apoyada en tic en la Institución Educativa Departamental Macondo. Cultura. Educación y Sociedad 9(3), 783-790. DOI: http://dx.doi. org/10.17981/cultedusoc.9.3.2018.92

\section{Resumen}

La investigación para muchas personas puede parecer un proceso tedioso, aburrido y sin ninguna importancia por la aparente complejidad del tema. Pero investigar permite el desarrollo de destrezas, habilidades y capacidades no desarrolladas por los estudiantes con la educación tradicional; al buscar alternativas para el desarrollo del pensamiento crítico, reflexivo y exploratorio sobre fenómenos que pasan en su vida cotidiana y como asociarlo a la teoría. El estudio tuvo como propósito implementar la investigación como estrategia pedagógica apoyada en tic en la institución educativa departamental Macondo. Este estuvo guiado bajo los lineamientos metodológicos de la IEP con un diseño basado en las trayectorias de indagación. La población estuvo conformada por cuarenta (40) estudiantes de básica primaria de la IED Macondo del municipio de Zona Bananera, Magdalena. Se logró una mejora en el proceso de enseñanza- aprendizaje con la implementación de la IEP apoyada en TIC puesto que se potencializaron aspectos como liderazgo, imaginación, capacidad reflexiva, pensamiento crítico, mejoramiento de habilidades sociales y creación de hipótesis.

Palabras clave: investigación como estrategia pedagogía, investigación, tecnologías de la información y la comunicación.

\section{Abstract}

Research for many people may seem a tedious, boring and unimportant process because of the apparent complexity of the subject. But research allows the development of skills, abilities and abilities not developed by students with traditional education; when looking for alternatives for the development of critical, reflective and exploratory thinking about phenomena that happen in their daily life and how to associate it with theory. The purpose of the study was to implement research as a pedagogical strategy supported by ICT in the Macondo departmental educational institution. This was guided under the methodological guidelines of the IEP with a design based on the trajectories of inquiry. The population consisted of forty (40) elementary school students from the Macondo IED of the municipality of Zona Bananera, Magdalena. An improvement in the teaching-learning process was achieved with the implementation of the IEP supported by ICT, since aspects such as leadership, imagination, reflexive capacity, critical thinking, improvement of social skills and creation of hypotheses were potentiated.

Keywords: research as pedagogy strategy, research, Technology of the information and communication.

1 Líder del grupo de investigación "Grupmacondo".

2 Docente de la institución educativa Macondo del municipio de la zona Bananera, departamento del Magdalena.

- The author; licensee Universidad de la Costa - CUC

Cultura, Educación y Sociedad vol. 9 no. 3, pp. 783-790. Diciembre, 2018

Barranquilla. ISSN 2389-7724 Online 


\section{Introducción}

La investigación puede ser una forma contundente de acercarse a las respuestas certeras o, al menos, satisfactorias, que nos conduzcan a la reflexión sobre diversos temas, tanto específicos como otros amplios, que pueden llegar a despertar la curiosidad y hasta el asombro. Si se desconoce un tema, se hace necesario investigarlo y con frecuencia sucede en la vida cotidiana que no prestamos atención a esta sentencia y corremos hacia el apresuramiento y la incertidumbre, sacando conclusiones apresuradas. Eso, en la vida escolar no debe suceder; el estudiante de hoy debe hacer uso de la investigación para satisfacer su apetito intelectual con resultados verificables o por lo menos con información analizada.

Colombia es un país en el cual las instituciones educativas hacen poco o ningún énfasis en enseñar a los educandos a investigar y la importancia de esto en su vida cotidiana. La mayoría de los docentes se encargan de dejar compromisos en los cuales los estudiantes deben indagar, pero la mayoría de estos solo acuden a internet donde solo con colocar una palabra surgen un sin número de respuestas donde la acción más frecuente es copiar y pegar.

El municipio de Macondo del departamento del Magdalena y sus instituciones educativas adscritas no son ajenos a esta problemática investigativa al demostrar sus estudiantes la apatía frente a la investigación, esto causado por la poca importancia que le dan las instituciones a este aspecto lo que provoca en el alumnado la falta de motivación ante este aspecto, poco conocimiento del tema, bajo desempeño por lo tanto en comprensión lectora ya que pocos leen y les basta solamente con la información que el docente les brinda en el aula de clases.

Por esto al planificar y ejecutar actividades para incentivar a los estudiantes a investigar los docentes están reestructurando y mejorando su práctica pedagógica a darle una función activa a los estudiantes dentro del proceso de aprendizaje y así dejar que estos investiguen temas que susciten de ellos mismos.

En la actualidad, existe una propuesta metodológica esta basados en la investigación, se desarrollan en los diferentes contextos y variados caminos con paradigmas corrientes, los cuales se inscriben propuestas basadas en el contexto de la investigación pedagógica. Dentro de esta teoría basada en investigación se encuentra la Enseñanza por descubrimiento y el Programa Ondas, con su propuesta de la Investigación como Estrategia Pedagógica (IEP), como una perspectiva que se desarrolla en ese enfoque y toma elementos de la pedagogía critico-liberadora y del enfoque socio-cultural (Mejía y Manjarrés, 2010; Herrera, Guerrero y Ramírez, (2018).

En ese mismo sentido, proceso educativo se convierten en problemas, y a partir de preguntas se estructura el currículo, en donde los avances de los estudiantes se determinan mediante la implementación de una estructura acorde a los conocimientos necesarios y autónomo, este guiado por el profesor, quien se encargará de una planificación enfocados en ejercicios y procedimientos para tal fin (Pozo, 2006; Ramírez \& Hugueth, 2017; Ramírez y Ampudia (2018). Este aprendizaje está fundado sobre ocho principios:

1. La capacidad para resolver problemas es la meta principal de la educación.

2. El entrenamiento en la heurística es mucho más importante que la entrega de de un contenido con significado. 
3. El método del descubrimiento es el camino para enseñar los contenidos curriculares.

4. Cuando el aprendizaje se hace por descubrimiento, ello organiza de manera eficaz lo aprendido.

5. El conocimiento verbal es la clave de cualquier transferencia.

En efecto, reconocer siempre que el proceso cognitivo tiene estas etapas nos permite recalcar que, en la investigación como estrategia pedagógica permite que se den una nueva constitución de culturas juveniles que trabajen de la mano con la sociedad, esta a su vez debe enfocar todos sus esfuerzos en construir en pensar mediante cambios cónsonos en una transformación direccionada por la investigación mediante a las tecnologías de la información y comunicación. En ese sentido, lo dicho has aquí supone que, en dinámica, el maestro y la maestra que trabajan con la esta herramienta tan valiosa como es la IEP comprenden una posibilidad de transformaciones que retroalimenten los distintos grupos sociales para producir cambios significativos en pertinencia a la práctica pedagógica haciendo real y concreta la idea.

Significa que, la propuesta no es formar científicos, es construir una cultura ciudadana y democrática en ciencia, tecnología e innovación para estos tiempos en un mundo construido sobre el conocimiento, la tecnología, la información y la comunicación. En otras palabras, el ejercicio pedagógico aparece como fundamento de una nueva forma de justicia educativa $y$ justicia curricular, actuando de forma coherente y justa con menos desigualdades en los niños, niñas y jóvenes, que optan por ser personas creativas, incursionando en un universo científico, como parte fundamental desde una propuesta metodológica fundamentada en la investigación.

El punto de partida para buscar estos caminos alternativos en la educación desde una perspectiva crítica se da a partir del reconocimiento en el trabajo inmaterial, tomando como base la sociedad como hecho generador de valores, elemento principal para construir mediante el conocimiento enfocado en la tecnología de la información actores que trabajen en pro de los nuevos procesos de conformación, basados en una sociedad entregada a estos cambios cónsonos en criterios que fundamentes un crecimiento sostenido investigativo (Mejía, 2011; Ramírez, Avendaño, Aleman, Lizarazo, Ramírez y Cardona, 2018; Rincón, Contreras \& Ramírez, 2017).

En efecto, pensar en la investigación desde corrientes educacionales sobre el conocimiento de la ciencia, la cultura, lo humano y su epistemología, enmarcan una acción que señalan la objetividad que permiten una construcción de formas pedagógicas que ayudan en generar formas de poder en la colectividad que a la postre servirán como bases sólidas para ayudar a las distintas instituciones en consolidar un proyecto educativo en pertinencia a investigación como una forma de control y la gestación de las desigualdades hoy en día existentes, y en estos tiempos, nuevas formas de acumulación y dominación (Ortega, 2009; Ramírez, Chacón y Valencia, 2018; Ramírez, Chacón y El Kadi, 2018).

En la esfera de la educación, así como en algunos grupos subalternos en el mundo del norte y en diferentes actores críticos de otros continentes, se ha desarrollado, en la perspectiva de los acumulados del paradigma latinoamericano, una concepción de trabajo edu- 
cativo que se caracteriza por ser una acción política, la cual busca transformar las condiciones de control, dominio y formas de sujeción de los actores, las comunidades e instituciones. Para lograrlo busca generar la conciencia crítica y las dinámicas sociales que impulsen que los grupos construyan formas de asociación y organización que los convierte en sujetos colectivos, constructores de su historia (Gadotti, 2003).

Así, las organizaciones, señalan Mendoza, López, \& Salas (2016), “son entes que generan sus propios objetivos y diseñan la forma como alcanzarlos teniendo en cuenta, necesariamente, las influencias del entorno social" (p. 62). Las escuelas no escapan de ello y es de allí que, en las organizaciones cualquiera que sea su ámbito, se hace preciso introducir una estratégica como como respuesta social (Mendoza, López y Salas, 2016)

De tal manera pues, que la investigación como estrategia debe ser concebida similar al pensamiento estratégico que según Arellano (2004) citado por Mendoza y López (2015), "hace referencia a la forma del pensamiento que se dirige hacia la creatividad, imaginación, y búsqueda de opciones innovadoras y realistas, como potenciación de lo posible enmarcado dentro de un proyecto de construcción intencional de la realidad" (p. 154).

En este entendido, exponen Hernández y Jiménez (2015), que estas redes "no reclama sólo la ética individual de las personas que componen las organizaciones, sino que ven un ente con responsabilidad propia económica, social y medioambiental a la que debe exigírsele comportamientos acordes a la misma" (p. 11). Según su razón de ser mediante las múltiples manifesta- ciones del poder en nuestra colectividad, esto con la intención en construir un espíritu de emancipación humana, para no desarrollar procesos de poder que controlan ni dominan, ni permitir que otros lo realicen en su propia vida (Torres, 2007).

Con lo cual "se podrá avanzar en el conocimiento sobre las condiciones de creación y de permanencia... de manera más amplia, del dinamismo del sistema social". (Maculan, Jiménez y Castellanos, 2015, p. 16). Va a ser el ejercicio de reconocer los contextos como lugares de saber. Por ello, busca devolver al acto educativo diferencia, heterogeneidad y multiplicidad fundadas en una diversidad cultural, social y cognitiva.

\section{Metodología}

La metodología del proyecto de investigación se realizó a partir de los recorridos de las trayectorias de indagación, basados en la integración de la IEP apoyada en TIC al aula, articulado al plan de estudios, desde las diferentes áreas se estructuraron temáticas relacionadas con la robótica educativa, la finalidad de este proceso investigativo, fue generar una trasformación social y educativa a través del aprendizaje en contexto, involucrando intereses, motivaciones y realidades de los estudiantes para generar conocimiento científico, donde se establecieron cinco (5) momentos que especifican en la ejecución y las metas trazadas para darle solución a la pregunta problema planteada. A continuación, se presentan los diferentes momentos.

- Momento (1): selección y análisis de la población sujeto de estudio.

- Momento (2): integración de la IEP apoyada en TIC al aula de clases, 
- Momento (3): socialización de los resultados de las indagaciones de estudiantes en una feria institucional dirigida por los docentes a cargo.

- Momento (4): análisis de los resultados de la implementación de la estrategia a través de la observación y diario de campo.

- Momento (5): propagación y divulgación de los resultados.

El proyecto se desarrolló en forma transversal, lo que quiere decir que se llevó a cabo en diversas asignaturas donde los docentes seguirán y aplicaban los pasos dados en la ruta metodológica determinada para cada caso con el fin de incentivar y acercar a los estudiantes a la investigación utilizando la misma como la estrategia metodológica aplicada.

Se utilizaron como fuentes de información múltiples herramientas que permitieron recolectar insumos para tener de primera mano información proporcionada por actores intervinientes en esta investigación, siendo estos los estudiantes en primera instancia los cuales en el desarrollo de sus clases se implementó la IEP, quienes brindaron aportes importantes para que luego lo docentes pudieran realizar el proceso de sistematización. Las fuentes de información fueron en su orden: la observación participante, la observación de campo y la encuesta cerrada.

Realizadas en ese orden con la finalidad de saber si esta estrategia genera o no un aprendizaje significativo en los estudiantes el desarrollo de actividades implementando la IEP. Al aplicar esta ruta metodológica en diferentes asignaturas se incentivó en los niños a investigar, haciendo uso de las TIC ya que este es un agente importante y novedoso para los estudiantes. En las investigaciones realizadas por los estudiantes se les dio la libertad de investigar acerca de las temáticas que observaran en el contexto en el que se encuentran inmersos.

Posteriormente se le aplicaron encuestas cerradas donde los niños respondieron si o no a los ítems encontrados en las mismas con la finalidad de medir que tanto se logró con la aplicación de las estas y si género en los estudiantes un aprendizaje significativo respecto a los temas tratados.

\section{Resultados}

De acuerdo con los razonamientos que se han venido realizando, el eje de esta propuesta está fundamentada que en la sociedad actual existen saberes netamente propios en referencia a una cultura, el cual establece de una forma permanente formas de conocimiento planeadas por grupos de jóvenes que busca la unidad mediante una relación de saberes y preparación en referente a los saberes como parte primordial a través de una propuesta pertinente a metodologías que permitan un conocimiento social integral en las diferentes concepciones, que podrán tramitar reconociendo la visibilidad de múltiples métodos investigativos, en coherencia con el tipo de problema que se trataba.

Los docentes lograron con la implementación de este proyecto que los estudiantes se motivaran más a investigar e indagar sobre temas de su interés, y que investigaran por gusto y no se sientan presionados, de esta forma se desarrolló un pensamiento crítico y reflexivo en ellos. Estas estrategias también alimentan en los estudiantes el deseo de seguir aprendiendo y ayudar a su comunidad trabajando en 
pro de disminuir problemáticas de su entorno inmediato. Por otra parte, con la implementación de las Tics dentro de la pedagogía consigue que las clases sean muy novedosas y salgan de lo tradicional como el libro, la libreta y el tablero.

\section{Conclusiones}

Se evidenció la vital importancia durante el desarrollo de este proyecto ya que los directos beneficiados fueron los estudiantes de la básica secundaria de las Instituciones Educativas Departamentales y por ende los docentes donde sus clases mejoraron notoriamente y al ser los estudiantes quienes investigan desde las situaciones más básicas pero que generaron interrogantes entre ellos y desde la aplicación de la IEP se aplicaron las rutas metodológicas propuesta por el programa Ciclón. Al realizar este proceso los estudiantes potencializaron aspectos como liderazgo, imaginación, capacidad reflexiva, pensamiento crítico, mejoramiento de habilidades sociales y creación de hipótesis entre otros.

Durante la elaboración de este proyecto se lograron proponer estrategias orientadas hacia la mejora del aspecto investigativo de los niños participantes, dejando en evidencia el interés por fomentar la formación integral de los estudiantes otorgando gran valor al sentido explorativo a través de actividades que promuevan la investigación dentro de los valores en las aulas de clases.

Por otro lado, haciendo uso de las TICS en estos procesos se pueden implementar ambientes virtuales de aprendizaje como entornos educativos mediados por la tecnología, en una construcción de saber y conocimien- to donde se encuentra la organización espaciotemporal y la disposición de los materiales, como también la distribución de los recursos didácticos, el manejo del tiempo y las interacciones que se dan en el aula. En ese sentido los ambientes virtuales de aprendizaje son espacios de socialización e interacción entre docente y estudiante, donde pueden poner en común el desarrollo de distintas actividades, como también su seguimiento y evaluación.

\section{Referencias}

Castro, C., Uribe, D. y Castro, J. (2014). Marco de referencia para el desarrollo de un sistema de apoyo para la toma de decisiones para la gestión de inventarios. INGE CUC, 10(1), 30-42. Recuperado de https://revistascientificas.cuc.edu.co/index.php/ ingecuc/article/view/340

Gadotti, M. (2003). Perspectivas actuales de la educación. Buenos Aires: Siglo XXI.

Herrera, B., Guerrero, H. y Ramírez, R. (2018). Investigación como estrategia pedagógica: Una mirada desde la educación, escuela y transformación de la comunidad global. Barranquilla: Editorial Universitaria de la Costa.

Hernández, H. y Jiménez, A. (2015). Responsabilidad social empresarial, zona urbana del distrito de $\mathrm{Ba}$ rranquilla: estudio etnográfico. Económicas CUC, 36(2), 9-18. Recuperado de https://revistascientificas. cuc.edu.co/economicascuc/article/ view/694/1

Fals-Borda, O. (1984). Resistencia en el San Jorge. Historia doble de la Costa. Tomo III. Bogotá, D.C.: Carlos Valencia Editores. 
Freire, P. (1986). Hacia una pedagogía de la pregunta. Conversaciones con Antonio Faúndez. Buenos Aires: La Aurora.

Maculan, A.-M., Jiménez, C. y Castellanos, O. (2015). Aprendizaje en el proceso de incubación de empresas de base tecnológica. Económicas CUC, 36(1), 15-48

Mariño, G. (2010). El diálogo en la educación de jóvenes y adultos. Dos propuestas pedagógicas para implementarlo. En, El taller dialógico/la recuperación de experiencias laborales. Bogotá, D.C.: OEI.

Mejía, M. y Manjarrés M. (2010). Las pedagogías fundadas en la investigación. Búsquedas en la reconfiguración de la educación. +Revista Internacional Magisterio, 42(1). 16 26.

Mejía, M. (2011). Las escuelas de la globalización. El conflicto por su reconfiguración. Bogotá, D.C.: Desde Abajo.

Mendoza, D. y López, D. (2015). Pensamiento estratégico: centro neurálgico de la planificación estratégica que transforma la visión en acción. Económicas CUC, 36(1),81-94. Recuperado de https://revistascientificas.cuc.edu.co/economicascuc/ article/view/669/26

Mendoza, D., López, D. y Salas, E. (2016). Planificación estratégica de recursos humanos: efectiva forma de identificar necesidades de personal. Económicas CUC, 37(1), 61-79. Recuperado de https://revistascientificas.cuc.edu.co/economicascuc/ article/view/1136/pdf_103

Ortega, P. (2009). Sujetos y prácticas de la pedagogía crítica. Bogotá, D.C.: El Búho.
Porlán, R. (1995). Constructivismo y escuela: hacia un modelo de enseñanza aprendizaje basado en la investigación. Sevilla: Diada.

Pozo, J. y. (2006). Aprender y enseñar ciencia. Madrid: Morata.

Ramírez, R. y Hugueth, A. (2017). Modelo de comunicación productiva para las organizaciones de salud pública en Venezuela. Revista Opción, 33(83). 305-335. Disponible en http://produccioncientificaluz.org/index.php/opcion/article/view/23118

Ramírez, R. y Ampudia, D. (2018). Factores de Competitividad Empresarial en el Sector Comercial. Revista Electrónica de Ciencia y Tecnología del Instituto Universitario de Tecnología de Maracaibo (RECITIUTM), 4(1). 16-32. Disponible en http://recitiutm. iutm.edu.ve/index.php/recitiutm/article/view/130

Ramírez, R., Avendaño, I., Aleman, L., Lizarazo, C., Ramírez, R. y Cardona, Y. (2018). Principles of social responsibility for the strategic management of the talent human public health organizations. Revista Espacios, 39(37). 22-27. Disponible en http://www.re$\mathrm{v}$ i s t a e s p a c i o s. c o m / a18v39n37/18393722.html

Ramírez, R., Chacón, H. y Valencia, K. (2018). Gestión del talento humano como estrategia organizacional en las Pequeñas y Medianas Empresas. $R e$ vista CICAG, 16(1): 20-42. Disponible en: http://ojs.urbe.edu/index.php/cicag/article/view/2929

Rincón, Y., Contreras, J. y Ramírez, R. (2017). Tendencias Emergentes para el desarrollo del Talento Humano. En, S. Tobón, J. Pérez, M. Serna y R. Loaiza. Las Competencias y la Gestión del Conocimiento. (320-338). Medellín: Editorial Corporación CIMTED. 
Ramírez, R., Chacón, H. y El Kadi, O. (2018). Gestión estratégica del talento humano en las PYMES. Medellín: Editorial Corporación CIMTED.
Torres, A. (2007). La educación popular. Trayectoria y actualidad. Bogotá, D.C.: El Búho. 